\title{
Towards the Validation of Noise Experiments in the CROCUS Reactor Using the TRIPOLI-4 Monte Carlo Code in Analog Mode
}

\author{
Oskari Pakari ${ }^{1}$, Davide Mancusi ${ }^{2}$, Odile Petit $^{2}$, \\ Andrea Zoia ${ }^{2}$, Vincent Lamirand ${ }^{1,3}$, and Andreas Pautz ${ }^{1,3}$ \\ ${ }^{1}$ Laboratory for Reactor Physics and Systems Behaviour (LRS) \\ Ecole polytechnique fédérale de Lausanne (EPFL), Lausanne, Switzerland \\ ${ }^{2}$ DEN-Service d'Etudes des Réacteurs et de Mathématiques Appliquées \\ (SERMA), CEA, Université Paris-Saclay, Gif-sur-Yvette, France \\ ${ }^{3}$ Nuclear Energy and Safety Division (NES) \\ Paul Scherrer Institut (PSI), Villigen, Switzerland \\ oskari.pakari@epfl.ch,vincent.lamirand@epfl.ch
}

\begin{abstract}
Intrinsic neutron noise experiments offer a non-invasive manner to measure the prompt decay constant or reactivity of fissile systems. Using the fluctuations in the density of fission chains, one can infer the kinetics parameters via correlation analysis such as the Rossi-alpha method. The models allowing for the interpretation of these measurements typically rely on the assumption of the system behaving according to point kinetics. When dealing with systems where point kinetics fail to predict the true time correlation - such as heterogeneous or large cores - the direct simulation of fission chains using Monte Carlo methods appears as the only reliable candidate to provide reference predictions for the correlation functions. Monte Carlo methods using explicit fission model libraries are thus being examined as tools for prediction in noise analysis. In this work we illustrate the developments and simulation results of the analog transport capabilities of the TRIPOLI4 Monte Carlo code coupled with the LLNL fission library FREYA, as applied to a set of neutron noise experiments carried out in the CROCUS zero-power reactor with emphasis on the identification of spatial effects. To validate the general capability of the code to predict noise correlations, we examine time distributions of the whole core fission and explicit detection reactions. We present the methodology to achieve a good agreement between experiments and simulations. We reproduced experimental results for relative $\alpha$, within typical biases, and conclude on the general feasibility of the analog method. We further explore a decoupled core model and analyze it using the noise method. The results indicate an effective method to treat decoupled systems.
\end{abstract}




\section{Introduction}

In this paper we illustrate the results of a collaboration between the Laboratory for Reactor Physics and Systems Behaviour (LRS) at EPFL and the Service d'Etude des Réacteurs et de Mathématiques Appliquées (SERMA) at CEA. Neutron noise measurements offer a non-invasive manner to determine the kinetics parameters of a zero-power system. The results of these measurements can be used to validate Monte Carlo (MC) methods designed to directly predict said values, such as standard k-static estimates of $k_{\text {eff }}$ or the iterated fission probability (IFP) for the kinetics parameters as used for example in the codes TRIPOLI-4 [1], MCNP6.2 [2] or Serpent 2 [3]. The use of analog MC transport, however, allows simulating the actual fission chains and as such takes explicitly the time fluctuations of neutron interactions into account. Noise measurements can be simulated directly by registering the time stamps of interactions of interest, notably $(\mathrm{n}, \mathrm{f})$ or observable detection reactions like (n,p) in Helium-3. By analyzing the time information, a user can hypothetically predict kinetics parameters or $k_{\text {eff }}$ without needing IFP, k-static or other common methods of prediction. Simulating neutron noise is key in several applications, encompassing multiplicity counting for safeguards and sub-critical reactivity assessment. The first attempts were realized with KENO-NR [4] using analog transport and power spectral density analysis methods. A newer MCNP-based code that incorporates correlation models for neutrons and photons as well as more realistic time treatment of emissions is MCNP-PoliMi[5] - validated for multiplicity experiments of $\mathrm{Pu}$-spheres and $\mathrm{UO}_{2}$ samples [6]. In light of possible applications to Accelerator Driven Systems [7], the study of sub-critical systems has attracted research efforts for noise methods in MC. Recently, a patched version of MCNP was used for KUCA pulse decay simulations [8]. We herein report on the recent developments of TRIPOLI-4 as a code for noise applications: We will describe the methodology, and contrast the simulation results to a set of sub-critical noise experiments carried out in the CROCUS reactor at EPFL. A wide range of sub-critical states were measured and analyzed using noise methods that offer new perspectives for MC code validation purposes [9], [10]. In this work we aim to expand on this previous knowledge. The overall goals are thus: I) Use TRIPOLI-4 to model a set of noise experiments carried out in the CROCUS zero-power reactor at EPFL; II) Establish a methodology on how to produce simulated noise data - by using the hypothesis that analog MC transport coupled to a fission library such as LLNL FREYA is required; III) Validate the methodology against experimental data and conclude on its applicability; IV) Probe 'exotic' reactor configurations for beyond point kinetics behavior.

\section{Neutron noise theory}

In fissile systems where thermal hydraulic and mechanical noise effects can be neglected, the observed neutron density fluctuations are referred to as zero-power noise. In this regime it is possible to observe detector counts originating from the same fission chain and thus exhibiting temporal correlations. Following [11], we denote the auto-correlation function of a point reactor, neglecting delayed neutrons:

$$
P_{i i}(\tau)=\epsilon F_{0}\left(Y_{1} e^{-\alpha|\tau|}+\delta(\tau)\right)
$$

The associated prompt amplitude $Y_{1}$ reads

$$
Y_{1}=\frac{\alpha \epsilon D_{\nu}}{2\left(\beta_{e f f}-\rho\right)^{2}},
$$


$\epsilon$ is the detector efficiency in counts $C$ over fission rate $F_{0}$, and $D_{\nu}$ is the Diven factor. We further introduce the prompt decay constant $\alpha$, the effective delayed neutron fraction $\beta_{\text {eff }}$, and the reactivity $\rho$. This formulation is also referred to as the single-exponent Rossi- $\alpha$ method, and is directly applicable to observable zero-power reactor noise using e.g. measurements of time interval distributions. Such a distribution, also referred to as Rossi distribution, follows an exponential shape with decay constant $\alpha$, exhibiting the prompt fission chain decay. Other methods to measure $\alpha$ include the Feynman- $\alpha$ and PSD methods, detailed e.g. in [11]. Multi-exponent models can take delayed neutrons or spatial effects into account - often by allowing for another decay constant and respective amplitude. Phenomena that can be described by multi-exponent models are e.g. a reflector source described in two-region models [12], higher prompt modes of $\alpha$ [13], and complex spatial behaviors [14]. As these effects tend to be of small magnitude in tightly coupled systems, they are often neglected, and the single-exponent model is used for fitting. Analog MC do not suffer from these assumptions and provide an unbiased estimate of correlations: as such analog $\mathrm{MC}$ represents the reference tool for simulation and prediction of neutron noise.

\section{TRIPOLI-4: Analog mode and the FREYA fission library}

Our code of choice for this work is TRIPOLI-4 (T4), a reference MC transport code used for criticality and shielding calculations [15]. By default, it uses implicit capture: since neutron histories are artificially kept alive, the local chain behavior is modified and could thus impact time correlations. Moreover, in default T4, the number of neutrons emitted per fission $\nu$ is determined by taking the integer part of the average $\bar{\nu}$ (which is provided by evaluated libraries), and then sampling the removed decimal fraction to decide whether to add another neutron. For the specific needs of analog transport simulation, T4 samples capture events explicitly and uses sophisticated fission libraries to sample a physically realistic number of fission neutrons, with their correlated angles and energies. For this work, we will use analog transport and the LLNL fission library FREYA for fission event treatment [16]. FREYA has been previously also coupled to MCNP [17], but to our best knowledge has not been validated against reactor noise experiments. We herein present a methodology making use of the T4 neutron track feature that was adapted for noise purposes. A coupling of the LLNL fission library with T4 has already been implemented and tested for a spontaneous fission source model [18]: in this work we present a first comparison and validation of this method for neutron noise problems.

\section{Design of a numerical experiment: CROCUS and TRIPOLI-4}

A series of sub-critical noise experiments were conducted in CROCUS to characterize the intrinsic noise field with emphasis on identifying, if any, spatial effects [19]. Spatial effects represent possible deviations of the observed reactor behavior with respect to point kinetics predictions. The sub-critical experiments consisted of a specific detector setup in the pursuit of forcing a potential non-point-kinetics behavior: two thin Helium-3 proportional counters were set into a cylindrical polyethylene block covered by a cadmium layer, thus first cutting the thermal flux and then moderating the flux reaching the detector. The two detection systems were set into the water reflector at mid-core height. Five different distances to the core $(4$ to $20 \mathrm{~cm})$ at five water levels $(800,850$, 900,925 and $950 \mathrm{~mm}$ ) were measured, totaling in 25 data sets. The analysis of the experimental results suggests a point reactor behavior. In order to confirm the experimental data, also in view of future efforts to use the noise field in perturbation studies, a direct analog MC simulation was 


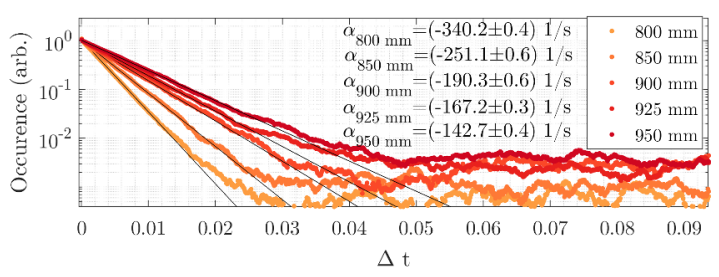

\section{Figure 1: Analog T4 + FREYA (n,f) time stamp Rossi distributions and fits for single exponent models for different water levels of CROCUS.}

performed. A ROOT geometry model of CROCUS was created for a benchmark on kinetics parameters [20], which is directly usable as input for T4. In the framework of the CORTEX H2020 program [21], the model was updated to match the COLIBRI [22], [23] fuel oscillation experiment; this reactor configuration is the experimental setup used for the neutron noise experiments. As a preliminary verification of the possibility of using analog $\mathrm{MC}$ transport to produce data comparable to experiments, we first focused on the tracking of every $(n, f)$ event. This is equivalent to having a detection system with perfect efficiency, i.e. 1 Count per Fission. We use the JEFF3.1.1 nuclear data library for all calculations. The simulated Rossi distributions corresponding to the five water level configurations (see Figure 1) follow the experimental trend. In order to produce validation data, we then also explicitly modeled the Helium-3 experiments. The tracking of events was done in post-processing using the ROOT-tracks feature of T4, which allows saving all neutron tracks, optionally filtered for interactions of interest (such as $(n, f)$ or $(n, p)$ ) to reduce the memory burden.

\subsection{Monte Carlo neutron noise methodology}

For the sake of conciseness, we summarized the methodology on how to create noise observables in Figure 2. The parameters that require tuning were identified as follows: 1) Noise analysis parameters, such as Rossi bin width or Feynman measurement time T; 2) Reactor configuration and simulation options such as the presence of delayed neutrons; 3) CPU time requirements; 4) source location, energy, angle, and intensity; 5) and finally the simulation type: Implicit capture, Analog, or Analog+FREYA. Our key findings are the following:

- To $\beta$ or not to $\beta$ : At first glance it might appear that delayed neutrons are necessary in order to allow for noise methods to yield physically meaningful results. In practice, suppressing the simulation of delayed neutrons reduces the reactivity of the system by $1 \$$, thus avoiding possible computational pitfalls close to criticality due to very long fission chains. We find that this reactivity difference does not affect the prompt decay constant inferred from the distributions. For the experiments considered herein, delayed neutrons might be completely ignored when assessing the multiplication of the system. Nonetheless, unless indicated otherwise, delayed neutrons are included in all further simulations.

- Computation time: A few hours of CPU time is enough to yield a rough estimate $(<20 \%)$ of $\alpha$ using $(\mathrm{n}, \mathrm{f})$ time stamps. 24 hours yield $<1 \%$ accuracy.

- The source problem: In k-static calculations, the inactive cycles are used to find the equilibrium fission source distribution. For sub-critical states, the k-static approach leads to a biased flux 


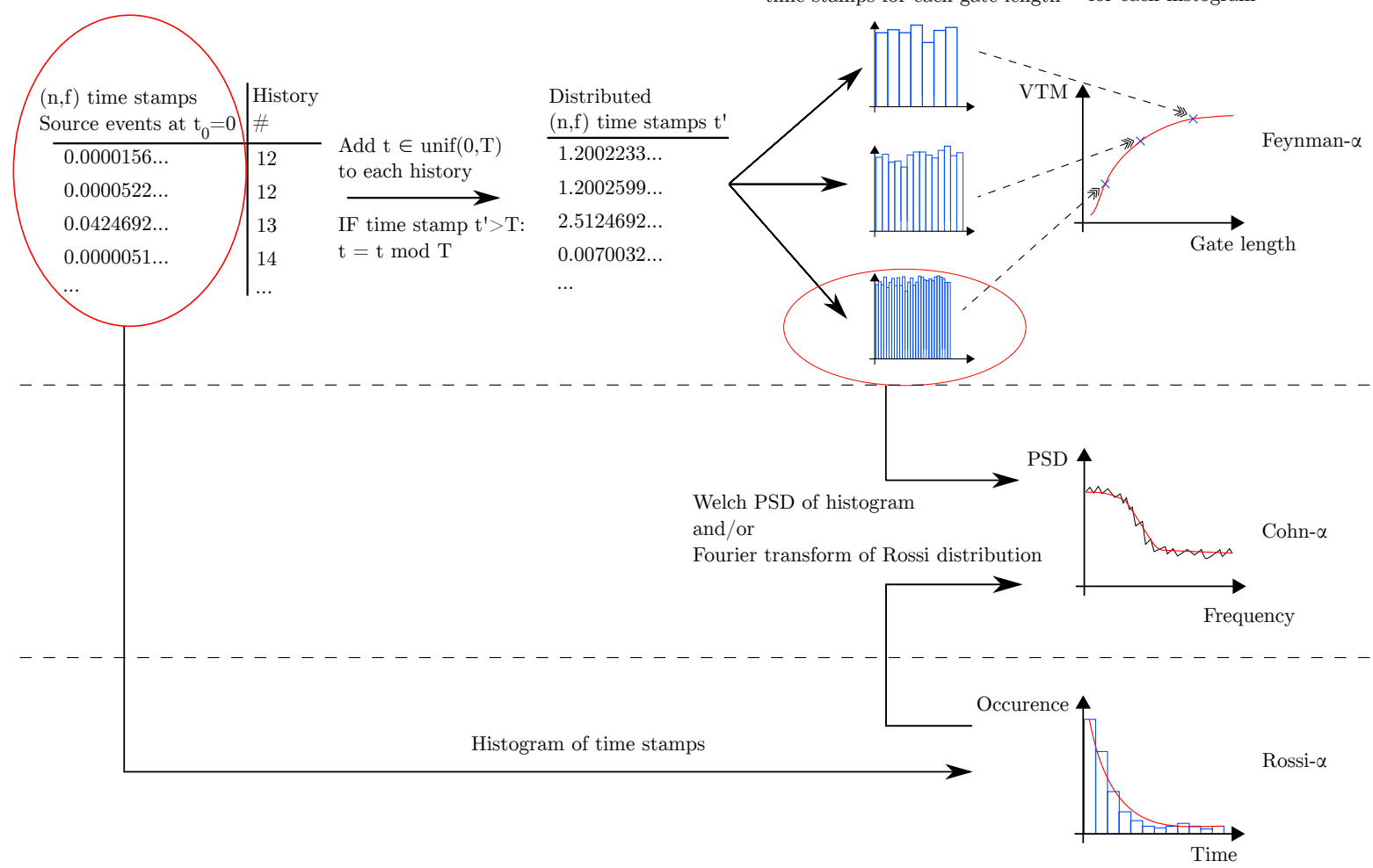

Figure 2: Illustration of how to treat time stamp data from MC simulations to create the observable noise data allowing for curve fitting with the Rossi, Feynman or PSD expressions.

shape*, as any sub-critical interrogation depends also on the source location and intensity. A uniform source distribution will underestimate the true fission chain length compared to a unbiased source. We observed that close to criticality $(950 \mathrm{~mm})$ the relative difference in $\alpha$ is about $3 \mathrm{pcm}$, while at $800 \mathrm{~mm}$ it is $\sim 35 \mathrm{pcm}$. This result is consistent with the fact that the source influence should diminish close to criticality. Also, it substantiates the hypothesis of fission chain lengths being dependent on the source location. Hence, for any sub-critical configuration the interrogation source location needs to be explicitly modeled to remove this bias ${ }^{\dagger}$.

\subsection{Comparison to experiments: $\alpha \boldsymbol{\&} \Delta \alpha$ for different configurations}

One might naively expect that the fitted Rossi- $\alpha$ values from analog calculations using FREYA agree with those measured in experiments. As a matter of fact, the fission chain length corresponds to the absolute reactivity $\rho_{\text {sim }}$ of the simulated system, and not the experimental value $\rho_{\text {exp }}$. Equivalently, IFP-method calculated kinetics parameters suffer from the same bias. Indeed, a

*Also, it leads to a biased estimation of $k_{\text {eff }}$ for systems far from criticality. "Far" is hereby very dependent on the geometry and materials of the problem [24].

${ }^{\dagger}$ In this work, the critical and sub-critical sources were post-processed to emit all neutrons at time zero. 
Table 1: Comparison of the prompt decay constant $\alpha$ for different water levels of CROCUS for experiments, IFP predicted values for Serpent 2 and T4, and T4 Noise. The fit results for

T4 noise using Rossi or Feynman distributions on full core (n,f) or detector (n,p) tracking are further differentiated by their simulation mode: implicit capture (IC), analog (A), and analog with FREYA fission $(\mathrm{A}+\mathrm{F})$. The uncertainties listed arise either from statistical estimates for the simulations or $95 \%$ confidence bounds on fits for the noise methods. We also compare relative $\Delta \alpha$ values with $800 \mathrm{~mm}$ as reference for the experiments, T4 Noise, and T4 IFP. The uncertainties are propagated from the aforementioned values.

\begin{tabular}{lccccc}
\hline Water level (mm) & 800 & 850 & 900 & 925 & 950 \\
\hline $\boldsymbol{\alpha}(\mathbf{1} / \mathbf{s})$ & & & & & \\
T4 IFP & $-340.8 \pm 2.6$ & $-253.9 \pm 1.5$ & $-186.2 \pm 2.3$ & $-152.1 \pm 2$ & $-128 \pm 2.2$ \\
Serpent 2 IFP & $-339.2 \pm 0.6$ & $-254.6 \pm 0.5$ & $-182.9 \pm 0.5$ & $-150.9 \pm 0.5$ & $-122.4 \pm 0.5$ \\
& & & & & \\
T4 Rossi A+F (n,f) & $-340.2 \pm 0.4$ & $-251.1 \pm 0.6$ & $-190.3 \pm 0.6$ & $-167.2 \pm 0.3$ & $-143.7 \pm 0.6$ \\
T4 Rossi A (n,f) & $-319.3 \pm 0.5$ & & & & $-134.2 \pm 0.9$ \\
T4 Rossi IC (n,f) & $-313.5 \pm 0.7$ & & & & $-149.9 \pm 4.3$ \\
T4 Rossi A+F (n,p) & $-340 \pm 10$ & $-259.9 \pm 6.4$ & $-179.9 \pm 5.4$ & $-178.9 \pm 6.3$ & $-144.6 \pm 7.7$ \\
Experiment [19] & $-378.2 \pm 3.0$ & $-301.3 \pm 1.2$ & $-232.5 \pm 0.7$ & $-201.7 \pm 0.8$ & $-174.9 \pm 0.8$ \\
Serpent 2 relative to 961.8 mm & $-389.9 \pm 0.8$ & $-305.3 \pm 0.7$ & $-233.6 \pm 0.7$ & $-201.6 \pm 0.7$ & $-173.1 \pm 0.7$ \\
& & & & & \\
$\Delta \boldsymbol{\alpha}$ to 800mm (1/s) & & & & & \\
Experiment & 0 & $76.9 \pm 3.3$ & $146 \pm 3.1$ & $177 \pm 3.2$ & $203 \pm 3.1$ \\
T4 Rossi A+F (n,f) & 0 & $89.1 \pm 1.6$ & $149.9 \pm 2.3$ & $173.3 \pm 2$ & $197.5 \pm 2.2$ \\
T4 IFP & 0 & $86.9 \pm 3.0$ & $154.7 \pm 3.5$ & $188.7 \pm 3.3$ & $212.8 \pm 3.4$ \\
\hline
\end{tabular}

consistent over-prediction of $k_{\text {eff }}$ by $\sim 230 \mathrm{pcm}$ is observed in the MC simulation CROCUS benchmarks with respect to the measured critical state [20]. A comparison with experiments is thus only possible by comparing relative decay constants or reactivities - e.g. as is common practice for rod worth estimations. Therefore, we will connect simulations to experiments only via relative decay constants:

$$
\Delta \alpha=\alpha_{\text {ref }}-\alpha_{\text {pert }},
$$

with $\alpha_{\text {ref,pert }}$ referring to arbitrary reference states that are compared to a "perturbed" state. In Table 1 we compare $\alpha$ for the experimental configurations as obtained with T4 using IFP ( $\alpha=$ $\left.\left(\rho_{\text {sim }}-\beta_{\text {eff }}\right) / \Lambda\right)$, T4 using noise methods and the Helium-3 experiments. The experiments are also contrasted to $\alpha$ obtained from Serpent 2 to provide code-to-code comparison. In addition, we display $\Delta \alpha$ for the experiments and T4 noise. Note that the reference for $\Delta \alpha$ is the respective value at $800 \mathrm{~mm}$. Our findings can be summarized as follows:

- The experiments agree with IFP predictions for $\alpha$ if the measured critical state $(961.8 \mathrm{~mm})$ is used as reference. However, the IFP $\alpha$ is also increasingly over-predicted with deeper subcriticality. This is consistent with the considerations presented in [24].

- Noise methods agree with IFP predictions for $\alpha$ only for sub-critical states $<900 \mathrm{~mm}$, differing by up to $15 \%$ closer to criticality. Using FREYA compared to pure analog or implicit capture reduces discrepancies of $\sim 100 \mathrm{pcm}$ to $<10 \mathrm{pcm}$. 


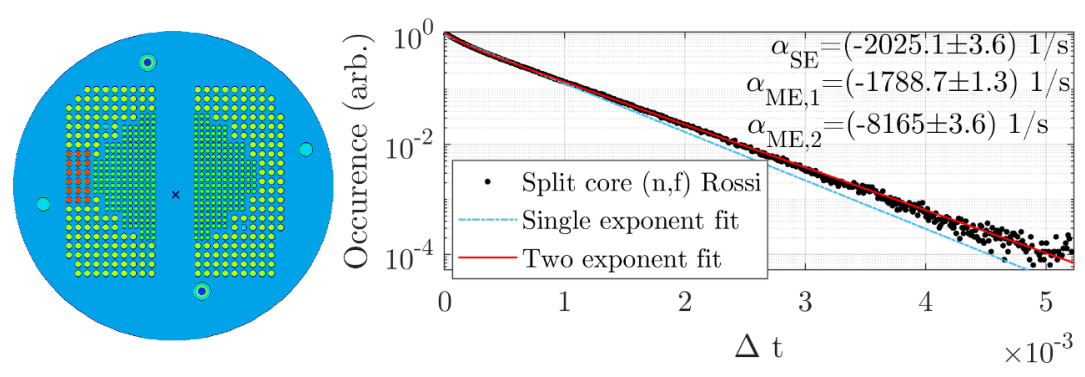

Figure 3: Left: T4 model of the CROCUS split core. Right: T4 (n,f) Rossi distribution with single (SE) and two (ME) exponent fits. The calculations used analog transport and FREYA.

- $(\mathrm{n}, \mathrm{f})$ and $(\mathrm{n}, \mathrm{p})$ agree within $1 \sigma$ to $2 \sigma$. The relative amount of time stamps per hour for $(\mathrm{n}, \mathrm{f})$ to $(\mathrm{n}, \mathrm{p})$ in Helium-3 should correspond to the experimental efficiency. Indeed, $(\mathrm{n}, \mathrm{f})$ tracking gives about $10^{6}$ time stamps per CPU hour, while (n,p) in a single detector gives $4.4 \times 10^{2}$ time stamps per hour. The ratio is $2.1 \times 10^{-4}$ - compared to the experimental efficiency of $1.1 \times 10^{-4}$ [19], we can infer an intrinsic efficiency of $\sim 50 \%$, a typical value for Helium-3 tubes. This finding further supports the soundness of our analysis.

- The difference between $\Delta \alpha$ confirms the hypothesis stated above: the absolute values are incompatible for comparison, unless the real critical state is used as reference - the relative prompt decay agrees with the experiments within $1 \sigma$, largely due to the experimental uncertainty. The values of $\Delta \alpha$ inferred from noise simulations accurately match experimental results.

\section{Exotic core configurations: Search for decoupled kinetics}

As an extended test of the capabilities of analog MC transport, we attempt to induce a departure from point kinetics behavior. Such a departure will likely exhibit two or more exponential decay constants in the Rossi- $\alpha$ distribution. Using the CROCUS model we thus conjectured that a spatial separation of the core into two fuel regions (see Fig. 3, left), could allow for such decoupled kinetics. For this purpose we examine again the Rossi- $\alpha$ distributions for $(n, f)$ in a modified CROCUS model split into two regions separated by $\sim 11.7 \mathrm{~cm}$, with a $k_{\text {eff }} \sim 0.92$. The source was chosen uniformly in the fissile volumes ${ }^{\ddagger}$. In Figure 3 we display the resulting Rossi- $\alpha$ distribution: indeed, a single exponential only captures the average prompt decay, while a two-exponential model more accurately yields two decay components: the larger at $\alpha_{2} \sim-81651 / \mathrm{s}$ (likely the decay constant in the respective core halves) and the smaller at $\alpha_{1} \sim-17881 / \mathrm{s}$ being the decay constant of both core halves when coupled.

Analog MC simulations can capture more complex behavior outside the realm of point kinetics likely more reliably than k-static calculations of decoupled systems. If $\beta_{\text {eff }}$ and $\Lambda_{\text {eff }}$ of the system are known, we would be able to infer half core and full core $k_{\text {eff }}$-values.

\footnotetext{
${ }^{\ddagger}$ We thus expect a bias when comparing to a localized source.
} 


\section{Conclusions}

In this paper we have discussed the methodology, numerical experiments, and comparison to experimental data of analog Monte Carlo simulations as applied to the interpretation of neutron noise experiments. Comparisons were performed with respect to a set of CROCUS sub-critical noise experiments. First, we have introduced a novel consistent methodology to calculate noise curves from Monte Carlo calculations. We briefly discussed the developed methodology and its implementation in T4 using analog transport, LLNL FREYA fission library and ROOT tracks features to create noise data. A more detailed analysis of the free parameters, such as binning of the Rossi histograms, source strength, CPU requirements, etc. will be presented in an extended version of this work. No correction factor was required to achieve agreement with experiments by comparing relative decay constants. The noise method allowed for the accurate prediction of $\alpha$. Using analog transport and FREYA impacts the noise curves significantly - in comparison to experiments, ana$\log$ transport with FREYA fission gives the best results. A split core model showed that decoupled problems can be also efficiently solved using analog MC simulation: this approach could be used in the design of loosely coupled systems, such as spent fuel repositories. Future research will be targeted at analyzing also the Feynman and PSD methods to predict $\beta_{\text {eff }}$ and explicitly modeling detectors and sources for decoupled problems.

\section{ACKNOWLEDGEMENTS}

This work has been partially supported by the ENEN+ project that has received funding from the EURATOM research and training Work Programme 2018 - 2019 - 1 \# 755576. TRIPOLI-4® is a registered trademark of CEA. We wish to thank Electricité de France (EDF) for partial financial support of the code TRIPOLI-4 $\mathrm{R}$.

\section{REFERENCES}

[1] G. Truchet, et al., Computing adjoint-weighted kinetics parameters in TRIPOLI-4® by the iterated fission probability method, Annals of Nuclear Energy 85 (2015) 17-26.

[2] C. Werner, et al., Mcnp6. 2 release notes, Tech. rep., Tech. Rep. LA-UR-18-20808, Los Alamos National Laboratory, Los Alamos, NM (2018).

[3] J. Leppänen, et al., Calculation of effective point kinetics parameters in the serpent 2 monte carlo code, Annals of Nuclear Energy 65 (2014) 272-279. doi:10.1016/j.anucene.2013.10.032.

[4] E. P. Ficaro, D. K. Wehe, KENO-NR: A monte carlo code for simulating 252cf-source-driven noise measurements to determine subcriticality, Nuclear Science and Engineering 117 (3) (1994) 158-176. doi:10.13182/nse94-a28531.

[5] S. Pozzi, et al., MCNPX-PoliMi for nuclear nonproliferation applications, Nuclear Instruments and Methods in Physics Research Section A: Accelerators, Spectrometers, Detectors and Associated Equipment 694 (2012) 119-125. doi:10.1016/j.nima.2012.07.040.

[6] S. Clarke, et al., Verification and validation of the MCNPX-PoliMi code for simulations of neutron multiplicity counting systems, Nuclear Instruments and Methods in Physics Research Section A: Accelerators, Spectrometers, Detectors and Associated Equipment 700 (2013) 135-139. doi:10.1016/j.nima.2012.10.025. 
[7] C. Rubbia, A high gain energy amplifier operated with fast neutrons, in: AIP Conference Proceedings, AIP, 1995. doi:10.1063/1.49069.

[8] A. Talamo, et al., Advances in the computation of the sjöstrand, rossi, and feynman distributions, Progress in Nuclear Energy 101 (2017) 299-311. doi:10.1016/j.pnucene.2017.01.006.

[9] O. Pakari, et al., Kinetic parameter measurements in the crocus reactor using current mode instrumentation, IEEE Transactions on Nuclear Science.

[10] O. Pakari, et al., Current mode neutron noise measurements in the zero power reactor crocus, in: EPJ Web of Conferences, Vol. 170, EDP Sciences, 2018, p. 04017.

[11] I. Pázsit, L. Pál, Neutron Fluctuations: A Treatise on the Physics of Branching Processes, Elsevier, 2007.

[12] G. D. Spriggs, et al., Two-region kinetic model for reflected reactors, Annals of Nuclear Energy 24 (3) (1997) 205-250.

[13] C. Berglöf, et al., Auto-correlation and variance-to-mean measurements in a subcritical core obeying multiple alpha-modes, Annals of Nuclear Energy 38 (2) (2011) 194-202.

[14] J. R. Sheff, R. W. Albrecht, The space dependence of reactor noise i-theory, Nuclear Science and Engineering 24 (3) (1966) 246-259.

[15] E. Brun, et al., TRIPOLI-4 $R$, CEA, EDF and AREVA reference monte carlo code, Annals of Nuclear Energy 82 (2015) 151-160. doi:10.1016/j.anucene.2014.07.053.

[16] J. Verbeke, et al., Fission reaction event yield algorithm FREYA 2.0.2, Computer Physics Communications 222 (2018) 263-266.

[17] J. Verbeke, et al., Integration of FREYA into MCNP6. an improved fission chain modeling capability., Tech. rep., Lawrence Livermore National Lab.(LLNL), Livermore, CA (United States) (2013).

[18] J. Verbeke, O. Petit, Stochastic analog neutron transport with TRIPOLI-4 and FREYA: Bayesian uncertainty quantification for neutron multiplicity counting, Nuclear Science and Engineering 183 (2) (2016) 214-228.

[19] O. Pakari, Experimental and numerical study of stochastic branching noise in nuclear reactors, Ph.D. thesis, École polytechnique fédérale de Lausanne (EPFL) (2020).

[20] A. Zoia, et al., Monte carlo analysis of the CROCUS benchmark on kinetics parameters calculation, Annals of Nuclear Energy 96 (2016) 377-388.

[21] C. Demaziere, et al., Overview of the CORTEX project, in: International Conference Physor '19, 2018.

[22] V. Lamirand, et al., The COLIBRI experimental program in the CROCUS reactor: characterization of the fuel rods oscillator, EPJ Web of Conferences 225 (2020) 04020. doi:10.1051/epjconf/202022504020.

[23] V. Lamirand, et al., Neutron noise experiments in the AKR-2 and CROCUS reactors for the european project CORTEX, EPJ Web of Conferences 225 (2020) 04023. doi:10.1051/epjconf/202022504023.

[24] D. E. Cullen, et al., Static and dynamic criticality: are they different?, Lawrence Livermore National Lab., Livermore, CA (US). 